\title{
Transitions with the benefit of Hindsight
}

Despite being a fundamental step in development, little is known about the mechanisms that regulate the switch from proliferation to differentiation. Work in the fruitfly now provides details of one such transition, which involves interactions of the Notch pathway with both cell-cycle regulators and Hedgehog (HH) signalling components.

The follicular epithelial cells that cover the Drosophila melanogaster germ line undergo mitosis until stage 6 of oogenesis, after which they stop proliferating, enter an endoreplication phase and begin differentiating. Notch signalling is needed for the switch from mitosis to endoreplication (the M-E switch), and the effects of Notch in this context are partly mediated by downregulation of the homeodomain gene cut, which itself controls a subset of cell-cycle regulatory proteins.
To identify other mediators of Notch function in the M-E switch, Sun and Deng did an antibodybased screen for proteins that show expression changes during the transition period and identified Hindsight (HNT), a zinc-finger transcription factor. Mutants that are defective for Notch signalling showed a loss of HNT expression in follicle cells, which also showed a reduction in the size of their nuclei, indicating an impaired M-E switch. So, Notch seems to upregulate HNT expression, which is needed for the transition out of proliferation. Consistent with this, the authors provided direct evidence that the $\mathrm{M}-\mathrm{E}$ switch is impaired in hnt mutants. Furthermore, misexpression of $h n t$ in follicle cells before stage 6 led to their premature entry into endoreplication.
How does hnt regulate the cell cycle to drive the M-E switch? Sun and Deng made flies with egg chambers that were mosaic for a hnt mutation, and found that the cell-cycle regulator CUT is continuously expressed in mutant cells, rather than being downregulated after stage 6 . The authors also showed that $h n t$ is required for the regulation of a second cell-cycle regulator, String, which provides a missing link between Notch signalling and the G2-M transition.

$\mathrm{HH}$ signalling is known to be involved in follicle-cell proliferation and, as the authors confirmed, components of this pathway are downregulated around the time of the M-E switch. Importantly, the authors showed that Notch signalling is required for this downregulation: in cells that
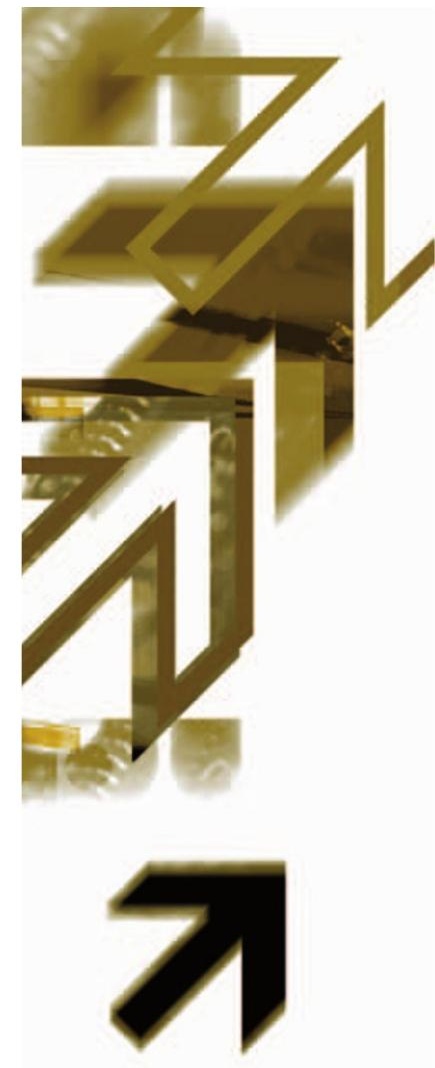

An eXpanding view of DNA methylation

DNA methylation is generally thought of as a mark of inactive chromatin. The recent finding that the active copy of the human $\mathrm{X}$ chromosome is more highly methylated overall than the inactive copy therefore comes as something of a surprise, prompting a rethink of how we view DNA methylation patterns.

One well-known characteristic of $\mathrm{X}$-chromosome epigenetics is that $\mathrm{CpG}$ islands at gene promoters are methylated on the inactive copy, contributing to gene silencing, and are unmethylated on the active X chromosome, allowing transcription. However, previous studies have hinted that, on a chromosome-wide scale, the active $\mathrm{X}$ chromosome might actually be more highly methylated.

To explore this possibility, Asaf Hellman and Andrew Chess developed a method for profiling the methylation status of sites across both the active and inactive $\mathrm{X}$ chromosomes ( $\mathrm{X} a$ and $X i)$. They made use of an array that allows 500,000 human singlenucleotide polymorphisms (SNPs) to be genotyped. By carrying out restrictionenzyme digestions that are sensitive to DNA methylation, followed by PCR and hybridization to the SNP array, the authors were able to determine allelespecific DNA methylation patterns across the $X$ chromosome. Active and inactive copies were distinguished by gene-expression and DNA-replication analyses, and DNA methylation patterns were compared in clonal cell lines, derived from a given individual, that had inactivated either the maternal or the paternal copy of the $\mathrm{X}$ chromosome.

The results showed a strikingly higher level of allele-specific DNA methylation on the $\mathrm{Xa}$ than on its inactive counterpart, at a ratio of about 2.4:1. Most of this methylation occurs within the bodies of genes (the transcribed regions), rather than in upstream promoters or intergenic areas. The authors found no biases that give a clue to the function of this methylation; for example, methylation was not especially common at tissuespecific genes or repetitive elements. Does this relative hypermethylation on the $\mathrm{X}$ a result from active methylation on this copy, or from demethylation of the Xi? To answer this question, Hellman and Chess looked at a human embryonic stem cell line, which is presumed to represent a stage in development before $X$ inactivation. Few of the Xa-specific methylation sites were methylated monoallelically in these cells, indicating that biallelic methylation is the initial state, with 
expressed a defective form of Notch, HH effector proteins were aberrantly expressed beyond stage 6 . By examining levels of these effector proteins in mosaic hntmutant egg chambers, Sun and Deng showed that HNT is required for the negative regulation of $\mathrm{HH}$ signalling by Notch. Furthermore, examination of the expression of cubitus interruptus, a key mediator of the HH signalling output, showed that HNT regulates the transcription of this gene.

This work in the fruitfly has clearly shown how interactions between major developmental signalling pathways, and their influence on the cell cycle, can trigger the switch between proliferation and differentiation. Future studies should clarify whether similar interactions have a role in other developmental contexts.

ORIGINAL RESEARCH PAPER Sun, J. \& Deng, W.-M. Hindsight mediates the role of Notch in suppressing Hedgehog signaling and cell proliferation. Dev. Cell 12, 431-442 (2007)

later demethylation of the $\mathrm{Xi}_{\mathrm{i}}$ at gene bodies.

The authors suggest a model for this differential DNA methylation pattern on the two copies of the $\mathrm{X}$ chromosome. They propose that inactive, untranscribed regions, such as the $\mathrm{Xi}$ and intergenic regions, are more likely to undergo loss of methylation, resulting in higher methylation levels remaining at gene bodies on the $\mathrm{Xa}$. Whether this is the case or not, this study alters our views of DNA methylation on the $X$ chromosome, revealing marked differences outside the promoter regions that have been the focus of previous studies and setting the scene for other reassessments of DNA methylation patterns.

Louisa Flintoft

ORIGINAL RESEARCH PAPER Hellman, A. \& Chess, A. Gene body-specific methylation on the active $\mathrm{X}$ chromosome. Science $\mathbf{3 1 5}$,

1141-1143 (2007)

WEB SITES

The Chess laboratory:

http://chgr.mgh.harvard.edu/chess/index.shtml

DEVELOPMENT

Chipping away at developmental networks

Over the years, classical genetic analysis has identified key regulators involved in metazoan development. With the advent of highthroughput approaches, the pathways are beginning to be assembled into networks, the topology of which provides further insights into how development is orchestrated. Two recent studies published in Genes \& Development used ChIP-on-chip experiments to elucidate transcriptional regulatory networks in the early development of Drosophila melanogaster, focusing on a few key transcription factors involved in the specification of mesoderm and the dorsoventral (DV) axis. They revealed unexpected crosstalk between biological processes and hitherto unappreciated modes of regulation.

Sandmann et al. focused on the central regulator of mesoderm development in D. melanogaster - Twist. To identify Twist-bound cis-regulatory motifs, the authors performed ChIP-on-chip analysis at two consecutive time periods in early fly development, combined with expression profiling of twist loss- and gain-offunction embryos. The difference in the binding of Twist to cis elements at these time points revealed the dynamic nature of Twist-mediated regulation. Furthermore, computational approaches followed by ChIP experiments found evidence for extensive combinatorial binding of Dorsal and Twist at the earlier time point and Tinman and Twist at the later time point.

The analysis unexpectedly revealed that Twist also regulates genes that are essential for proliferation and transcriptional regulation. The authors combined the temporal binding map of Twist with in vivo binding data for Myocyte enhancing factor 2 (MEF2), Dorsal and Tinman three key mesodermal transcription factors. The resulting core network of transcriptional regulation of early mesoderm development reveals extensive combinatorial binding and frequent feed-forward regulation. The topology of this network argues against the hierarchical model of a master regulator function. Instead of regulating only a few factors, each of which has a handful of their own direct targets, Twist directly affects multiple levels in this developmental network.

Zeitlinger et al. also used ChIP-on-chip analysis but their focus was on genome-wide discovery of the targets of the DV determinants: Dorsal and its two earliest targets, Twist and Snail. Their ChIP-on-chip analysis was performed on embryos in which Toll receptor has been constitutively activated, which leads to high levels of Dorsal, Twist and Snail, and makes all of the embryo's cells adopt a mesodermal fate. As expected from the genome-wide scale of this work, new DV enhancers were identified. But the discovery that many segmentation genes contain binding sites for Dorsal, Twist and/or Snail uncovered previously unanticipated links between the DV and anterioposterior (AP) networks. Moreover, binding sites for these transcription factors were also found at genes that encode components of signal transduction such as Decapentaplegic (DPP), Epidermal growth factor (EGF) and Notch pathways.

Together, the studies show how ChIP-on-chip analysis can extend the core sets of regulators that are known from genetic studies. In doing so, they reveal previously unanticipated insights into the architecture and the extent of connectivity of early developmental networks.

Magdalena Skipper

ORIGINAL RESEARCH PAPERS Sandmann, T. et al. A core transcriptional network for early mesoderm development in Drosophila melanogaster. Genes Dev. 21, 436-449 (2007) | Zeitlinger, J. et al. Whole-genome ChIP-chip analysis of Dorsal, Twist and Snail suggests integration of diverse patterning processes in the Drosophila embryo. Genes Dev. 21, 385-390 (2007) FURTHER READING Kimelman, D. Mesoderm induction: from caps to chips. Nature Rev. Genet. 7, 360-372 (2006)

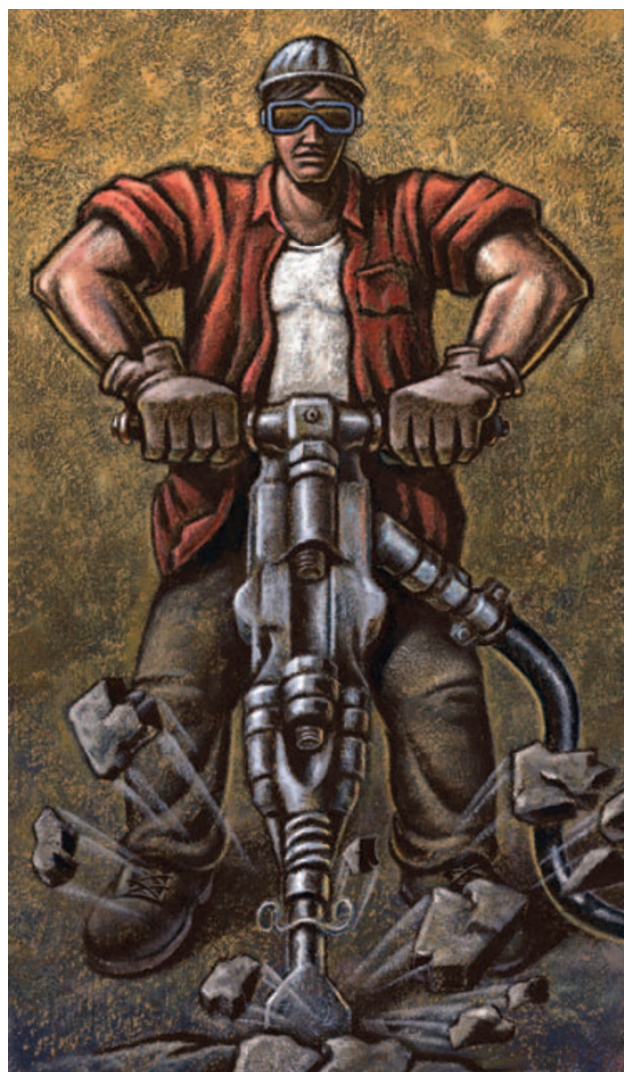

